\title{
sensors
}

ISSN 1424-8220

(C) 2007 by MDPI

www.mdpi.org/sensors

Full Research Paper

\section{Physical-Mechanical Properties of Nitrodopes Affected by Ultra-Violet Radiation}

\author{
Suzana Cakić $^{1}$, Ljiljana Raskovic ${ }^{2}$, Časlav Lačnjevac ${ }^{3, *}$, Milos Rajkovic ${ }^{3}$, Miroljub Barać ${ }^{3}$ \\ and Miodrag Stojanovic ${ }^{4}$
}

1 Faculty of Technology, 16000 Leskovac, Bulevar oslobodjenja 124, Serbia. E-mail: Suzana Cakic, suzana_cakic@yahoo.com

2 Pomoravlje, Nis, Serbia. E-mail: Ljiljana Raskovic, duda@bankerinter.net

3 Faculty of Agriculture, 11000 Belgrade, Nemanjna 6, Serbia. E-mail: Časlav Lačnjevac, ukilaki@eunet.yu; Miroljub Barać,baracm@agrifaculty.bg.ac.yu; Milos Rajkovic, rajmi@agrifaculty.bg.ac.yu

4 VS-VTI, 11000 Belgrade, Serbia. E-mail: Miodrag Stojanovic, stveljko@ptt.yu

* Author to whom correspondence should be addressed.ukilaki@eunet.yu or lcaja@agrifaculty.bg.ac.yu

Received: 11 June 2007 / Accepted: 3 October 2007 / Published: 9 October 2007

\begin{abstract}
The FTIR spectroscopy has been employed in this research work to monitor the process of nitrodope photodegradation, by measuring surfaces of bands typical of a nitro group. Nitric esters are subject to degradation, which is reflected on a quantitative ratio of the surfaces of the IR bands that originate from the nitric ester. The obtained results show that the length of the UV rays' activity on the samples over the time periods of 240, 480 and 960 minutes directly affects the spectrum appearance of the same sample before and after the irradiation. The longer the action time of the UV rays and the higher a mass percentage of nitrocellulose in the nitrodope is, the smaller the bands' surfaces become, i.e. the level of degradation is higher. In order to confirm the degradation of nitrodope, the degree of crosslinking has also been examined by determining the König hardness and also the mean viscosity molar mass has been defined repeatedly applying the capillary viscosimetry method.
\end{abstract}

Keywords: UV-radiation, nitrodope, nitrocellulose, FTIR spectroscopy, König hardness 


\section{Introduction}

Synthetic macromolecular compounds are widely applied in practice and their usage is increasingly wider from one year to the next. They are used in the production of plastic materials, synthetic fibres, paints, varnishes, and electroinsulating materials, in the car industry, space industry etc [1].

Industrial nitrocellulose (to be more exact cellulose nitrate, since ester is involved) is one of the oldest and fairly used substances, which is deposited in the form of film. Although this substance has been known to exist for more than a thousand years, until the end of World War I it had mostly been used for the manufacture of celluloid, photo films, and artificial leather, and in the industry of paints and varnishes to produce nitrodope and nitrocombination enamels. Its distinctive characteristics were manifested to the full extent only after the production of low viscosity types had been developed, which made it possible to increase the dry matter content in a film and to reduce the needed number of paint coatings.

At the same time the development of high viscosity types has taken place thus giving rise to the new era in the industry of varnishes. The oil varnishes that had been used by that time revealed defects, primarily caused by slow drying. The drying takes place chemically by bonding the oxygen from the air. This process is unnecessarily prolonged even after the hardening of a film, consequently resulting in its cracking. In contrast to this, the drying process in nitrodopes is short and it develops in an entirely physical way by evaporation of solvent and diluent. Dry films of nitrodopes show great elasticity and stability to various physical and chemical effects such as temperature changes, moisture, alcohol, dilute acids etc.

The higher drying rate of nitrodopes has enabled to bring assembly lines into the industry of furniture and other industrial branches causing the rapid rise in labour productivity. Owing to all told, the use of industrial nitrocellulose has become considerably wider, and nitrocellulose has become a basic component of varnishes for wood and metals, of coatings for paper, foils and films as well as in the production of printing paints and adhesives. In recent years, against strong competition due to the rapid development in the field of synthetic materials, nitrocellulose has continued to exist as a substance that is deposited in the form of film in a lot of industrial branches [2].

\subsection{Degradation}

There are a large number of reactions that lead to aggravation of polymer properties. By this is, in the first place, meant the reactions of breaking bonds in macromolecules, in which process the products of considerably lower molar mass are obtained.

These reactions are referred to as degradation reactions and they develop affected by heat, light, ionizing radiation, oxygen, ozone, mechanical stress etc. The degradation reactions are generally undesired since they result in deterioration of physical mechanical and other properties of polymers, too. Thus, for example, contrary to the crosslinking reaction, the degradation reaction leads to the formation of a polymer of a lower molar mass, which stipulates the abrupt deterioration of the mechanical properties and enables the occurrence of the flow at lower temperatures [2]. 


\subsection{Photodegradation}

The degradation in polymers may also arise under the effect of light (UV rays); this process is named the photodegradation. Depending on the wavelength of the UV rays, that is, on their energy, the breaking off of bonds between side atoms or groups and the basic chain of a macromolecule may occur, as well as the breaking of the bonds within the basic chain of the macromolecule [1].

Resulting from the action of the light rays on a polymer, from the breaking of chemical bonds and the formation of active centres-radicals, the chain process of degradation may develop as well as the integration of the monomer molecules, depending on the conditions under which the given polymer is. Surely, as the temperature increases, so does the degree of photodegradation. The degree of photodegradation is dependent on the polymer structure, the wavelength of the UV- rays and the conditions under which the polymer is.

In view of the fact that during the photodegradation the breaking of the primary, chemical, bonds in the macromolecules occurs, it is clear that the degree of photodegradation will depend on the strength of these bonds, that is, on the composition and the structure of the polymers. If the bonds are weaker, they break off more easily because, under all other equal conditions, smaller energy is required and vice versa.

In the process of the photodegradation, the effect of the wavelength of the UV-light is also essential. The weaker the bonds that are being broken off are, the smaller the energy of the UV rays is required, i.e., their wavelength is greater ( $\mathrm{E}$ and $\lambda$ are inversely proportional magnitudes ).

The enhancement of the light intensity accelerates the photodegradation reaction. Decomposition of polymers affected by UV- light is also known as the photolysis [1;3].

\subsection{Photodegradation of nitrocellulose}

With certain products, especially in paint coatings, the UV- radiation as a prime cause of molecular decomposition is very significant. The photodegradation is a much more complex process because radiation induces not only the primary degradation, but it also activates the products of degradation bringing them to a state of higher energy, which may give rise to a start of the autocatalytic reaction [2].

It has been known for a long time that the nitrocellulose is subject to photochemical degradation. As far back as 1867 , the exposure of the nitrocellulose to powerful light was noticed to lead to a gradual loss of important physical properties and eventually to the complete degradation. Since thermal degradation slightly contributes to instability of the nitrocellulose at room temperature, it is significant to estimate and understand the effect of the photochemical degradation under these conditions. Even the diffused laboratory light decomposes the nitrocellulose seriously over time, particularly if it is dissolved.

The research on the photochemistry of the nitrocellulose has advanced much slowlier than the studies of the thermal degradation of the nitrocellulose, partly on account of the lack of interest of scientists and partly because it is difficult to carry out these experiments. The use of the nitrocellulose in the industry of varnishes has provoked the interest in this problem. In the scope between $410 \mathrm{~nm}$ and $200 \mathrm{~nm}$ (from the violet to the far UV-range) the absorption curve of the nitrocellulose, containing 
from $10.9 \%$ to $14.1 \%$ of nitrogen, is continually increasing without reaching the maximum value [4, $5]$.

In the near UV (350 nm), hydroxyl groups are more subject to absorption than the nitric groups, whereas the reversely holds for the shorter wavelengths.

Based on the obtained results, it has been found that the nature of the reaction depends on the radiation frequency. Short wavelengths mainly induce the viscosity change without a considerable loss of nitrogen, whereas greater wavelengths cause a heavier loss of nitrogen and a minor change in viscosity. The two mechanisms that occur during the decomposition of nitrocellulose by means of the UV- light are the same as the models of the thermal degradation. The loss of nitrogen with a slight alteration of viscosity, taking place at the great wavelengths, is caused by the decomposition of the nitric ester. The very same mechanism is used to explain the thermal degradation at higher temperatures. The studies on simple alkyl nitrates have shown that three primary processes develop in the near UV range:

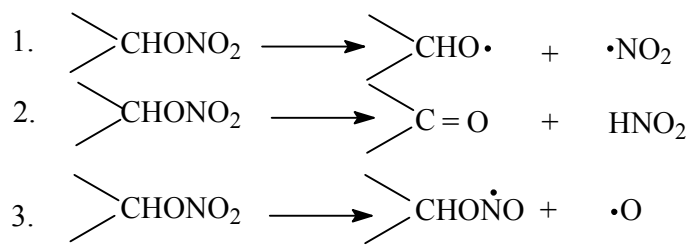

The first process is favored. The reaction continues in the following way:

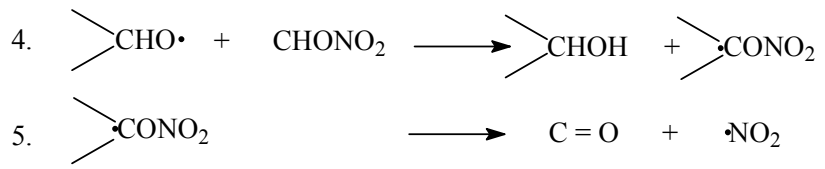

This mechanism results in the loss of nitrogen, but not also in the shortening of the chain. On the other hand, the shorter wavelengths of the UV range cause the alteration in the viscosity along with a minor change in the nitrogen content.

Irradiation of solid nitrocellulose by the UV-light of $290 \mathrm{~nm}$ induces a quicker loss of nitrogen from the nitrocellulose with the substitution degree of 1.9 than from the nitrocellulose with the substitution degree of 2.6.

A polymer with the substitution degree of 1.9 mainly contains glucose dinitrate. The mechanism of ester dissociation dominates and gives rise to the reduction of the nitrogen/carbon ratio. The mechanism of the ring disintegration can arise only in the rings that contain three nitric esters, as in the nitrocellulose with the substitution degree of 2.6 , and it leads to a slight loss of nitrogen $[3 ; 6]$.

\subsubsection{Mechanisms of Primary Decomposition}

Low temperature studies provide a basis for redefining cellulose nitrate decomposition into three different mechanisms. The first is conventional acid-catalyzed ester cleavage. The second involves a nitrogen-oxygen bond cleavage. This is the predominant mode of thermal decomposition above $100^{\circ} \mathrm{C}$, or when the molecule is irradiated with visible and near UV light and its occurrence is not dependent on the number of nitrate ester groups on the ring. Both of these decomposition pathways 
are supported extensively in the chemical literature. The third mechanism is much more speculative and arises from findings of unique events under special conditions. These include a different activation energy for thermal breakdown at lower temperatures and regimes where molecular weight changes rapidly but nitrogen content does not. This breakdown occurs photochemically in the far ultraviolet where nitrate ester groups absorb and not in the near ultraviolet where they do not. In any event, it occurs only on rings bearing three nitrate groups, a situation that cannot be explained by the other two mechanisms, and so at least one more has to be invoked. The data best fit a very improbable spontaneous coming apart of one entire nitrated glucoside ring within the polysaccharide chain. Therefore, the primary mechanisms for cellulose nitrate decomposition are: (1) acid catalyzed ester cleavage, (2) homolytic scission of the nitrogen-oxygen bond, and (3) ring disintegration.

\subsubsection{Secondary Modes of Decomposition}

Since it is not possible to eliminate or suppress all the primary mechanisms, the question of secondary reactions must be considered. At the very least, the slow thermal mechanisms provide traces of nitrogen oxides that will accumulate with time. The autocatalytic effect of this buildup needs to be studied and defined.

It is apparent from the results of many investigations on the mechanism of decomposition of nitrocellulose that this decomposition takes place in two stages. In the first stage, the reaction is relatively slow; in the second stage, the initial decomposition products react with the nitrocellulose, and the reaction becomes autocatalytic and therefore accelerates rapidly. If the products of decomposition in the first stage are removed as rapidly as possible (for example, by a stabilizer), the reactions of the second stage are prevented, and the decomposition is held down to a relatively slow rate.Decomposition of a nitrate ester at elevated temperatures is, in many respects, similar to the breakdown of an organic hydroperoxide. Both initially go through homolytic cleavage between heteroatoms to form oxy radicals. The oxy radicals in each case give both ketone and alcohol in a ratio that depends to a large extent on the availability of protons in the environment. Ketone may be the minor product in the cellulose nitrate system but there is evidence that further decomposition goes through the ketone and not the hydroxyl group. Ketones generally exist in tautomeric equilibrium with an unsaturated, enolic isomer and in a cellulose nitrate ring this configuration probably predominates. Olefinic molecules are reaction sinks for reactive species like ozone and nitrogen oxides. Addition occurs rapidly to form highly oxidized, reactive, and unstable species that readily undergo decomposition. The nature of secondary reactions at ambient temperatures is more speculative. Little is known about the chemistry that is occurring under conditions where ring disintegration is the predominant reaction. It is known that $\mathrm{NO}_{2}$ is an initial decomposition product and is rather easily converted to NO.

Oxygen in the short term does not enhance decomposition [7]. By taking these facts into account a mechanism of secondary decomposition can be formulated as shown in Figure 1. An initially formed oxy radical converts to a cyclic ketone; this conversion step may be the source of NO formation. The keto-enol structure is relatively stable but in the presence of $\mathrm{NO}$ and $\mathrm{NO}_{2}$ addition reactions occur. These lead to products that contain multinitrated rings, with dinitrated carbons. These are structures that should be highly susceptible to ring disintegration. Therefore, trinitrated rings can 
be expected to be less stable than dinitrated rings by secondary modes of decomposition at ambient conditions.
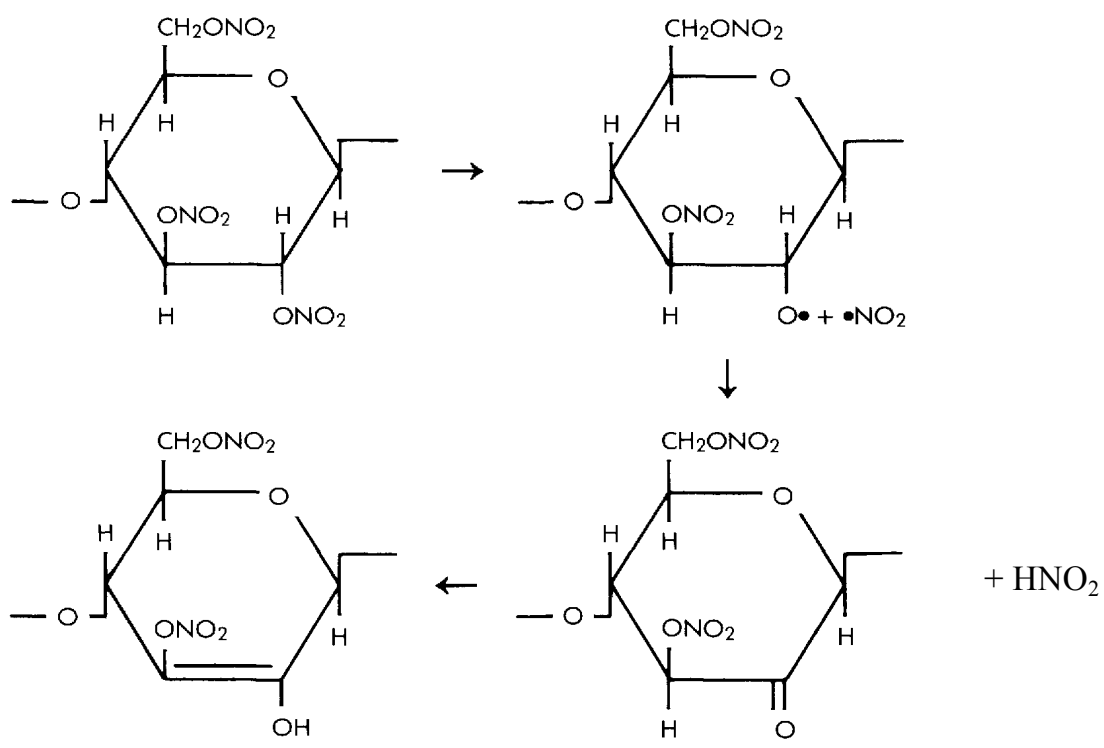

$\bullet \mathrm{NO}_{2} \downarrow$

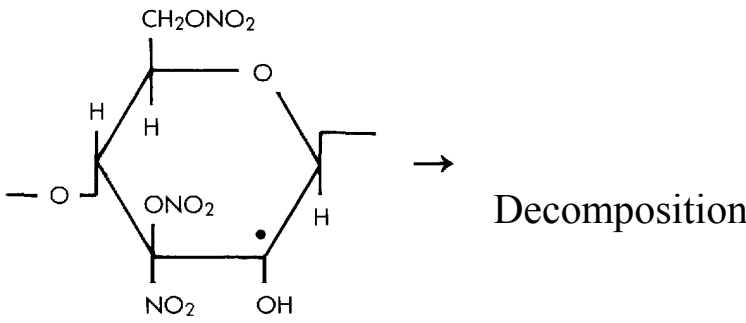

$\bullet \mathrm{NO}_{2} \downarrow$

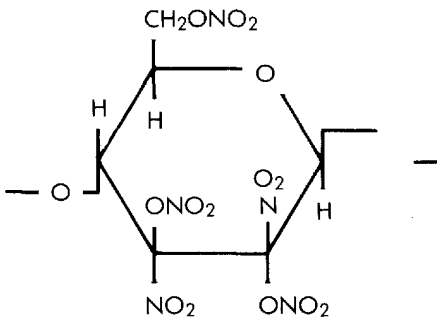

Decomposition

Figure 1. Pathways of secondary decomposition.

There is additional enhancement of secondary modes of decomposition by ultraviolet. Photolysis gives $\mathrm{NO}$ and atomic oxygen and, in the presence of molecular oxygen, the reactions indicated in equations 6 to 12 can occur: 


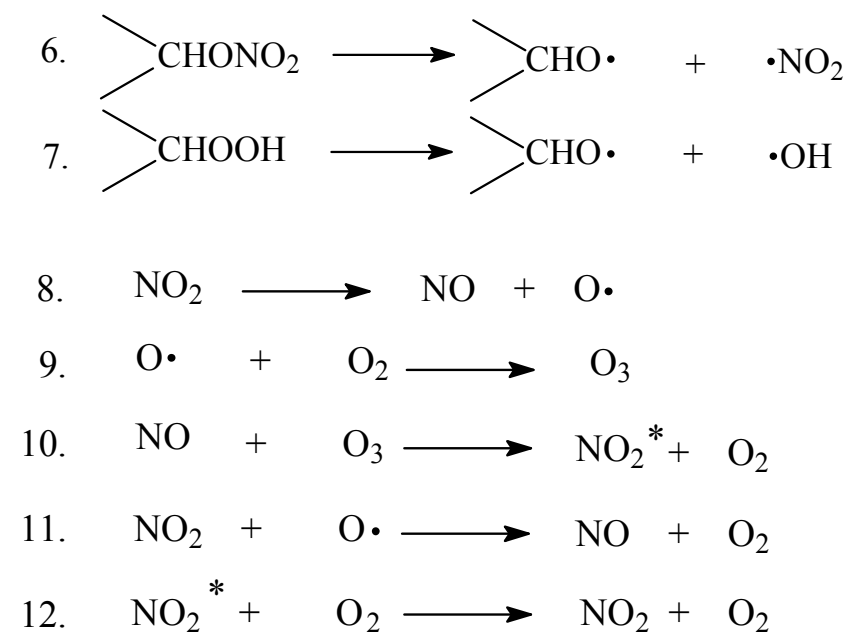

In particular the reaction between ozone and NO is highly exothermic and leads to electronically excited $\mathrm{NO}_{2}$. Thus, the effect of UV radiation is to enhance markedly the decomposition caused by secondary reactions particularly where the radiation takes place in the presence of oxygen. Table 1 indicates what happens to the viscosities of solution of samples of cellulose nitrate that are: (a) kept dark; (b) exposed to UV under nitrogen, and (c) exposed to UV in air for 200 hours. Clearly the additional presence of oxygen dramatically accelerates the light-induced decomposition [8].

Table 1. The effect of UV light on cellulose nitrate stability.

\begin{tabular}{|l|c|c|c|c|c|}
\hline \multirow{2}{*}{ Condtionon } & \multicolumn{2}{|c|}{ Relative Viscosity } & \multicolumn{2}{c|}{ Intrinsic Viscosity } \\
\cline { 3 - 6 } & Start & Finish & Start & Finish \\
\hline a. & Dark, air & 1,266 & 1,258 & 1,187 & 1,031 \\
\hline b. & $\mathrm{UV}, \mathrm{N}_{2}$ & 1,268 & 1,231 & 1,179 & 1,148 \\
\hline c. & $\mathrm{UV}$, air & 1,270 & 1,031 & 1,195 & 0,153 \\
\hline
\end{tabular}

Primary processes appear to be slow and allow a tolerable period of stability; ultimately, secondary processes are destructive.There is, however, an initial time period when secondary reactions are also slow and polymer integrity is essentially maintained. There is a need, therefore, to know and understand how quickly secondary rates increase as products accumulate and how far the polymer can go into the secondary decomposition time period - once this is added on to the primary decomposition time period - and still maintain critical properties. There are two methods that should be used to accomplish this end. One basic approach to arriving at a better understanding of at least the preliminary phases of the secondary chemistry is to use ultrasensitive analytical procedures that can measure very small changes under ambient conditions in short periods of time.

\section{Experimental}

Samples have initially been prepared as a mixure of nitro diluent, n-butylalcohol, butyl acetate and dioctylphthalate. Nitrocellulose (15\%) had been added to the mixture whereupon the homogenization 
lasting 10 minutes was performed. Upon the additon of the nitrocellulose, the resins AZALCHID RD $25 \mathrm{~T}$, AZALCHID S 37 and POLICOL $\mathrm{N}$ were added to the mixture and the homogenization was carried out once again. The time period needed to perform this homogenization was a bit longer (about 20 minutes) than the previous one due to the presence of the added viscous resins.

Subsequently, nitrocellulose with the increasing mass percentages was added to the mixture and the samples were deposited on glass plates where the formation of nitrodope dry films took place. The dry films were being exposed to UV radiation over the time periods of 240, 480 and 960 minutes while their physical-mechanical properties before and after the exposure were being studied.

Table 2. Prescription content of colourless nitrodope.

\begin{tabular}{|l|c|c|c|c|}
\hline Component & $\begin{array}{l}\text { Sample I } \\
\text { mass } \%\end{array}$ & $\begin{array}{l}\text { Sample II } \\
\text { mass } \%\end{array}$ & $\begin{array}{l}\text { Sample III } \\
\text { mass \% }\end{array}$ & $\begin{array}{l}\text { Sample IV } \\
\text { mass \% }\end{array}$ \\
\hline Nitro diluent & 36.8 & 36.8 & 36.8 & 36.8 \\
\hline n-butylalcohol & 7.0 & 7.0 & 7.0 & 7.0 \\
\hline Butyl acetate & 17.0 & 17.0 & 17.0 & 17.0 \\
\hline Dioctylphthalate & 4.0 & 4.0 & 4.0 & 4.0 \\
\hline${ }^{1}$ Nitrocellulose WOLFF & 15 & 18 & 20 & 25 \\
\hline $\begin{array}{l}{ }^{2} \text { AZALCHID RD 25 } \\
\text { (Alkyd resin) }\end{array}$ & 5.0 & 5.0 & 5.0 & 5.0 \\
\hline $\begin{array}{l}{ }^{3} \text { AZALCHID S 37 } \\
\text { (alkyd resin) }\end{array}$ & 10.0 & 10.0 & 10.0 & 10.0 \\
\hline $\begin{array}{l}\text { POLICOL N } \\
\text { (phenol-formaldehyde resin) }\end{array}$ & 3.0 & 3.0 & 3.0 & 3.0 \\
\hline
\end{tabular}

The process of the nitrocellulose degradation has been examined by the FTIR spectroscopy using the BOMEM Hartmann \& Braun MB-100 spectroscope, within the range of wave numbers between 4000 and $400 \mathrm{~cm}^{-1}$ at the resolution of $2 \mathrm{~cm}^{-1}$.

The FTIR spectra of the colourless nitrodope have been obtained by the method of KBr-pills (150 $\mathrm{mg} \mathrm{KBr}$ and $1 \mathrm{mg}$ sample). Nitrocellulose can be identified by the characteristic IR band of the nitric group at $1660 \mathrm{~cm}^{-1}\left(v_{\mathrm{as}}\right)$ and $1280 \mathrm{~cm}^{-1}\left(v_{\mathrm{s}}\right)[10]$.

The samples of the colourless nitrodope with different mass percentages of the WOLFF nitrocellulose have been irradiated by a UV lamp (Helling Super Light C 10 A) of the wavelength $\lambda=$ $365 \mathrm{~nm}$ and over the time periods of 240, 480 and 960 minutes.

The determination of König hardness of varnish films has been performed by the method (JUS H. C. 8,055), which dictates to define hardness in an indirect way, by measuring the time span in which the amplitude of oscillation of the pendulum, leant on a dried up polymer film, becomes smaller. 
For the purpose of testing in accordance with this standard, the plates sized $100 \times 100 \times 5 \mathrm{~mm}$, made of flat and smooth glass have been used. The mean value of the results obtained from three successive measurements, expressed in seconds, represents the hardness of a film [4].

The mean molar mass of nitrodopes, before and after the exposure to UV- rays, has been determined by the method of capillary viscosimetry, applying the Kun-Mark-Houvink-Sakurad equation:

$$
[\boldsymbol{\eta}]=\mathbf{K} \cdot \mathbf{M}_{\eta}{ }^{\alpha}
$$

where $\mathrm{K}$ and $\alpha$ are the constants for a given polymer - solvent system at a given temperature. For the solution of nitrocellulose in acetone $\mathrm{K}=2.5310^{-4}, \alpha=0.795$.

\section{Results and Discussion}

\subsection{Degree of nitrodope degradation monitored by determining the König hardness}

The hardness values of the dry films with different nitrocellulose mass percentages, before and upon the irradiation by a UV lamp over the periods of 240, 480 and 960 minutes are shown in Table 3.

Table 3. The hardness values of the nitrodope dry films before and after the action of UV rays.

\begin{tabular}{|c|c|c|c|c|}
\hline \multirow{2}{*}{$\begin{array}{c}\text { Action time } \\
\text { of UV-rays } \\
\text { (min.) }\end{array}$} & \multicolumn{4}{|c|}{ König hardness (") } \\
\cline { 2 - 5 } & Sample I & Sample II & Sample III & Sample IV \\
\hline 0 & 118 & 162 & 179 & 182 \\
\hline 240 & 129 & 154 & 169 & 176 \\
\hline 0 & 140 & 176 & 185 & 192 \\
\hline 480 & 166 & 166 & 178 & 181 \\
\hline 0 & 133 & 170 & 175 & 188 \\
\hline 960 & 148 & 169 & 172 & 182 \\
\hline
\end{tabular}

Table 3 shows that only in the samples with the lowest percentage of nitrocellulose (I) the degree of crosslinking has not decreased. In all other samples with the higher percentage of nitrocellulose, upon 240, 480 and 960- minute action of the UV-rays the decrease of the crosslinking degree, i.e. the degradation, has occurred which indicates that the photolysis has led to the breaking of bonds in macromolecules causing the deterioration of the physical-mechanical properties of the polymers $[7,8]$.

The process of decomposition affected by the UV-rays has been examined through the alteration of the molar mass of the samples before and after the irradiation. Tables $4-7$ exhibit the viscosity values of dilute solutions of the nitrodope dry films dissolved in acetone. Based on them, the diagrams of $\boldsymbol{\eta}_{\mathrm{sp}} / \mathrm{c}$ dependence on the solution concentracion have been drawn and are shown in Figure 2. The outflow time of the solvent, that is acetone, is $\mathrm{t}_{0}=23.5$ seconds. 
Table 4. Viscosity values of nitrodope solution in acetone for sample I.

\begin{tabular}{|c|c|c|c|c|}
\hline Sample I & \multicolumn{4}{|c|}{ No action of UV-rays } \\
\hline $\begin{array}{l}\text { Solution } \\
\text { concentration,C (\%) }\end{array}$ & $\mathbf{t}_{\mathbf{s r}}(\mathbf{s})$ & $\boldsymbol{\eta}_{\text {rel }}=\mathbf{t} / \mathbf{t}_{\mathbf{0}}$ & $\boldsymbol{\eta}_{\mathbf{s p}}=\boldsymbol{\eta}_{\text {rel }}-1$ & $\boldsymbol{\eta}_{\mathbf{s p}} / \mathbf{C}$ \\
\hline 0,25 & 25,0 & 1,06383 & 0,06383 & 0,25532 \\
\hline 0,50 & 26,5 & 1,12766 & 0,12766 & 0,25532 \\
\hline 0,75 & 28,5 & 1,21277 & 0,21277 & 0,28369 \\
\hline & \multicolumn{5}{|c|}{ After 240-minute action of UV-rays } \\
\hline 0,25 & 24,5 & 1,04255 & 0,04255 & 0,17021 \\
\hline 0,50 & 25,8 & 1,09787 & 0,09787 & 0,19574 \\
\hline 0,75 & 27,0 & 1,14894 & 0,14894 & 0,19858 \\
\hline & \multicolumn{5}{|c|}{ After 960-minute action of UV-rays } \\
\hline 0,25 & 24,1 & 1,02553 & 0,02553 & 0,10213 \\
\hline 0,50 & 25,0 & 1,06383 & 0,06383 & 0,12766 \\
\hline 0,75 & 26,5 & 1,12766 & 0,12766 & 0,17021 \\
\hline
\end{tabular}

Table 5. Viscosity values of nitrodope solution in acetone for sample II.

\begin{tabular}{|c|c|c|c|c|}
\hline \multicolumn{1}{|c|}{ Sample II } & \multicolumn{4}{|c|}{ No action of UV-rays } \\
\hline $\begin{array}{l}\text { Solution } \\
\text { concentration,C(\%) }\end{array}$ & $\mathbf{t}_{\text {sr }} \mathbf{( s )}$ & $\boldsymbol{\eta}_{\text {rel }}=\mathbf{t} / \mathbf{t}_{\mathbf{~}}$ & $\boldsymbol{\eta}_{\text {sp }}=\boldsymbol{\eta}_{\text {rel }} \mathbf{~}$ & $\boldsymbol{\eta}_{\text {sp }} / \mathbf{C}$ \\
\hline 0,25 & 26,5 & 1,12766 & 0,12766 & 0,51064 \\
\hline 0,50 & 28,1 & 1,19574 & 0,19574 & 0,39149 \\
\hline 0,75 & 29,6 & 1,25957 & 0,25957 & 0,34610 \\
\hline \multicolumn{5}{|c|}{ After 240-minute action of UV-rays } \\
\hline 0,25 & 26,2 & 1,11489 & 0,11489 & 0,45957 \\
\hline 0,50 & 27,2 & 1,15745 & 0,15745 & 0,31489 \\
\hline 0,75 & 29,6 & 1,25957 & 0,25957 & 0,34610 \\
\hline \multicolumn{5}{|c|}{ After 960-minute action of UV-rays } \\
\hline 0,25 & 25,0 & 1,06383 & 0,06383 & 0,25532 \\
\hline 0,50 & 26,0 & 1,10638 & 0,10638 & 0,21277 \\
\hline 0,75 & 26,9 & 1,14468 & 0,14468 & 0,19291 \\
\hline
\end{tabular}


Table 6. Viscosity values of nitrodope solution in acetone for sample III

\begin{tabular}{|c|c|c|c|c|}
\hline \multicolumn{1}{|c|}{ Sample III } & \multicolumn{4}{|c|}{ No action of UV-rays } \\
\hline $\begin{array}{l}\text { Solution } \\
\text { concentration,C(\%) }\end{array}$ & $\mathbf{t}_{\mathbf{s r}} \mathbf{( s )}$ & $\boldsymbol{\eta}_{\text {rel }}=\mathbf{t} / \mathbf{t}_{\mathbf{0}}$ & $\boldsymbol{\eta}_{\text {sp }}=\boldsymbol{\eta}_{\text {rel }} \mathbf{~}$ & $\boldsymbol{\eta}_{\text {sp }} / \mathbf{C}$ \\
\hline 0,25 & 27,8 & 1,18298 & 0,18298 & 0,73191 \\
\hline 0,50 & 29,0 & 1,23404 & 0,23404 & 0,46809 \\
\hline 0,75 & 31,2 & 1,32766 & 0,32766 & 0,43688 \\
\hline & \multicolumn{5}{|c|}{ After 240-minute action of UV-rays } \\
\hline 0,25 & 26,8 & 1,14043 & 0,14043 & 0,56170 \\
\hline 0,50 & 28,1 & 1,19574 & 0,19574 & 0,39149 \\
\hline 0,75 & 30,0 & 1,27660 & 0,27660 & 0,36879 \\
\hline & \multicolumn{5}{|c|}{ After 960-minute action of UV-rays } \\
\hline 0,25 & 25,9 & 1,10213 & 0,10213 & 0,40851 \\
\hline 0,50 & 26,9 & 1,14468 & 0,14468 & 0,28936 \\
\hline 0,75 & 28,0 & 1,19149 & 0,19149 & 0,25532 \\
\hline
\end{tabular}

Table 7. Viscosity values of nitrodope solution in acetone for sample IV

\begin{tabular}{|c|c|c|c|c|}
\hline \multicolumn{1}{|c|}{ Sample IV } & \multicolumn{4}{|c|}{ No action of UV-rays } \\
\hline $\begin{array}{l}\text { Solution } \\
\text { concentration,C(\%) }\end{array}$ & $\mathbf{t}_{\text {sr }}(\mathbf{s})$ & $\boldsymbol{\eta}_{\text {rel }}=\mathbf{t} / \mathbf{t}_{\mathbf{0}}$ & $\boldsymbol{\eta}_{\text {sp }}=\boldsymbol{\eta}_{\text {rel }}-\mathbf{1}$ & $\boldsymbol{\eta}_{\text {sp }} / \mathbf{C}$ \\
\hline 0,25 & 28,7 & 1,22128 & 0,22128 & 0,88511 \\
\hline 0,50 & 30,0 & 1,27660 & 0,27660 & 0,55319 \\
\hline 0,75 & 31,9 & 1,35745 & 0,35745 & 0,47660 \\
\hline & \multicolumn{4}{|c|}{ After 240-minute action of UV-rays } \\
\hline 0,25 & 27,5 & 1,17021 & 0,17021 & 0,68085 \\
\hline 0,50 & 29,0 & 1,23404 & 0,23404 & 0,46809 \\
\hline 0,75 & 30,1 & 1,28085 & 0,28085 & 0,37447 \\
\hline & \multicolumn{4}{|c|}{ After 960-minute action of UV-rays } \\
\hline 0,25 & 26,6 & 1,13191 & 0,13191 & 0,52766 \\
\hline 0,50 & 27,6 & 1,17447 & 0,17447 & 0,34894 \\
\hline 0,75 & 28,0 & 1,19149 & 0,19149 & 0,25532 \\
\hline
\end{tabular}




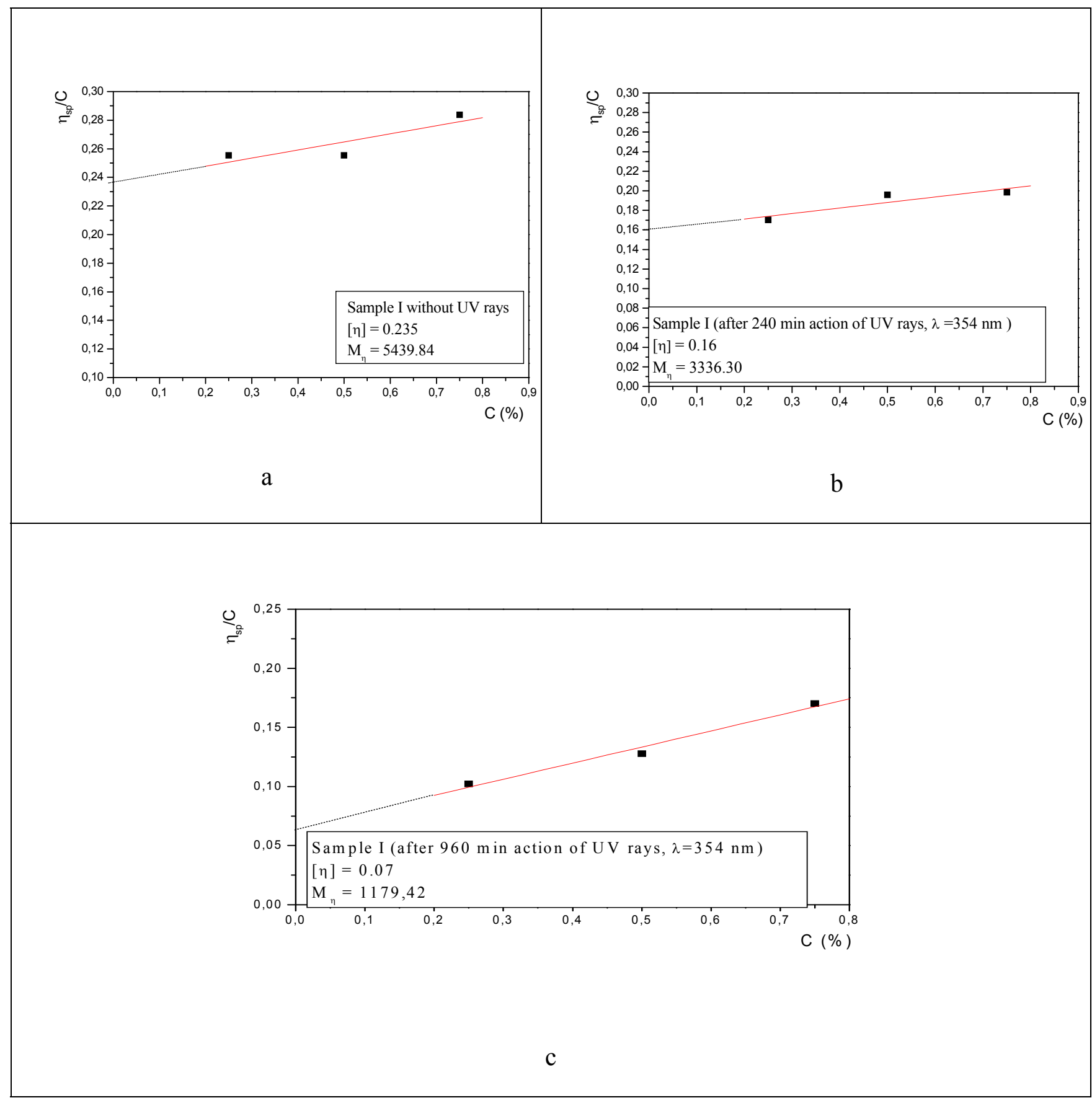

Figure 2. Dependence of reduced viscosity on the concentration of nitrodope dissolved in acetone, for sample I : a) no action of UV-rays, b) after 40-minute action of UV-rays, c) after 960-minute action of UV-rays.

Figure 3 shows the mean molar mass values of nitrodope samples with different mass percentages of nitrocellulose, before and after the irradiation that have been determined by using the values of a limiting viscosity number obtained by measuring the outflow time of nitrodope dissolved in acetone. 


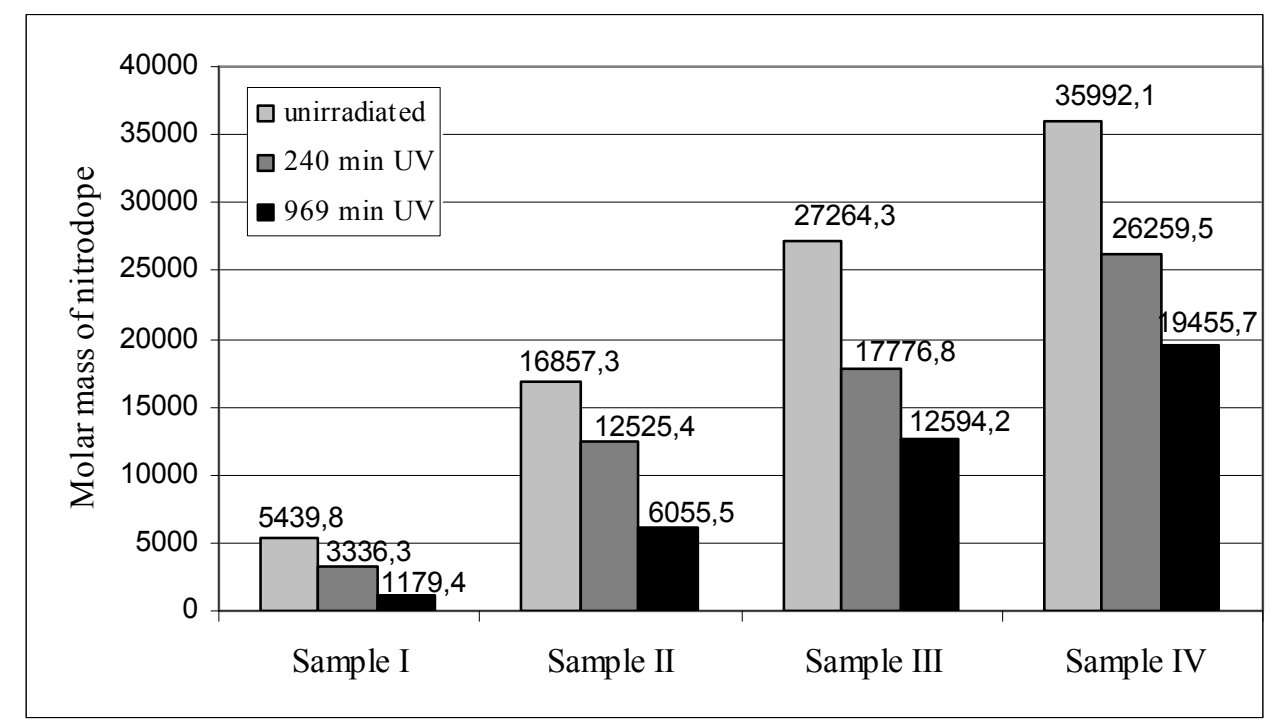

Figure 3. Collective graph of the dependence of the mean molar mass of nitrodope on the mass percentage of nitrocellulose before and after the UV radiation.

Based on the diagram in Figure 2 and the collective graph in Figure 3, it can be observed that the limiting viscosity $[\eta]$ and the mean molar mass $M_{\eta}$ rise with the increase of the nitrocellulose mass pecentage in a sample, and that their values decrease after the UV radiation, that is, the longer the radiation time period the lower the values of $[\eta]$ and $M_{\eta}$ are $[8 ; 9]$.

The decrease of the mean molar mass value after the radiation is mostly distinctive in the sample with the highest content of nitrocellulose (IV) and it is directly proportional to the length of the exposure.

This indicates that the value decrease of the limiting viscosity $[\eta]$ and the mean molar mass $M_{\eta}$ after the action of the UV-rays is the result of the degradation caused precisely by the effect of the UV-light of the $365 \mathrm{~nm}$ wavelength. This is because the degradation induces the breaking of ester bonds whereat the formation of a polymer with a lower value of the mean molar mass takes place, which may produce the deterioration of the polymer's mechanical properties and the occurrence of flow at lower temperatures [8].

\subsection{Degree of nitrodope degradation monitored by the FTIR spectroscopy}

In studying the degradation processes affected by the UV radiation, special attention has been paid to the research on the ranges of spectra in which valence vibrations of the nitric group occur, specifically at the wave numbers of $1660 \mathrm{~cm}^{-1}$ (asymmetric valence vibration) and of $1280 \mathrm{~cm}^{-1}$ (symmetric valence vibration). During the nitrocellulose degradation by UV-rays the decomposition of the nitric ester takes place [10;11]. It is observed through the decline in intensity of the typical IR bands that occurs at the wave numbers of $1660 \mathrm{~cm}^{-1}$ and $1280 \mathrm{~cm}^{-1}$ and is shown in Figure 4.

The FTIR spectra given in Figure 4 clearly indicate the intensity decline of the typical IR bands of the nitric group at the wave numbers of $1280 \mathrm{~cm}^{-1}\left(v_{\mathrm{s}}\right)$ and $1660 \mathrm{~cm}^{-1}\left(v_{\mathrm{as}}\right)$. The intensity of the characteristic bands is the highest prior to the irradiation of the samples by the UV-light [4]. 
This reduction of the surfaces of the IR bands is a characteristic of the nitric ester photodegradation under the effect of UV-rays, that is, it confirms the first primary process of the photodegradation. The obtained results point to the reactions of breaking the ester bonds in a macromolecule itself, which may result in aggravation of the physical-mechanical properties as well as in the formation of polymers with the lower molar mass $[6,2]$.

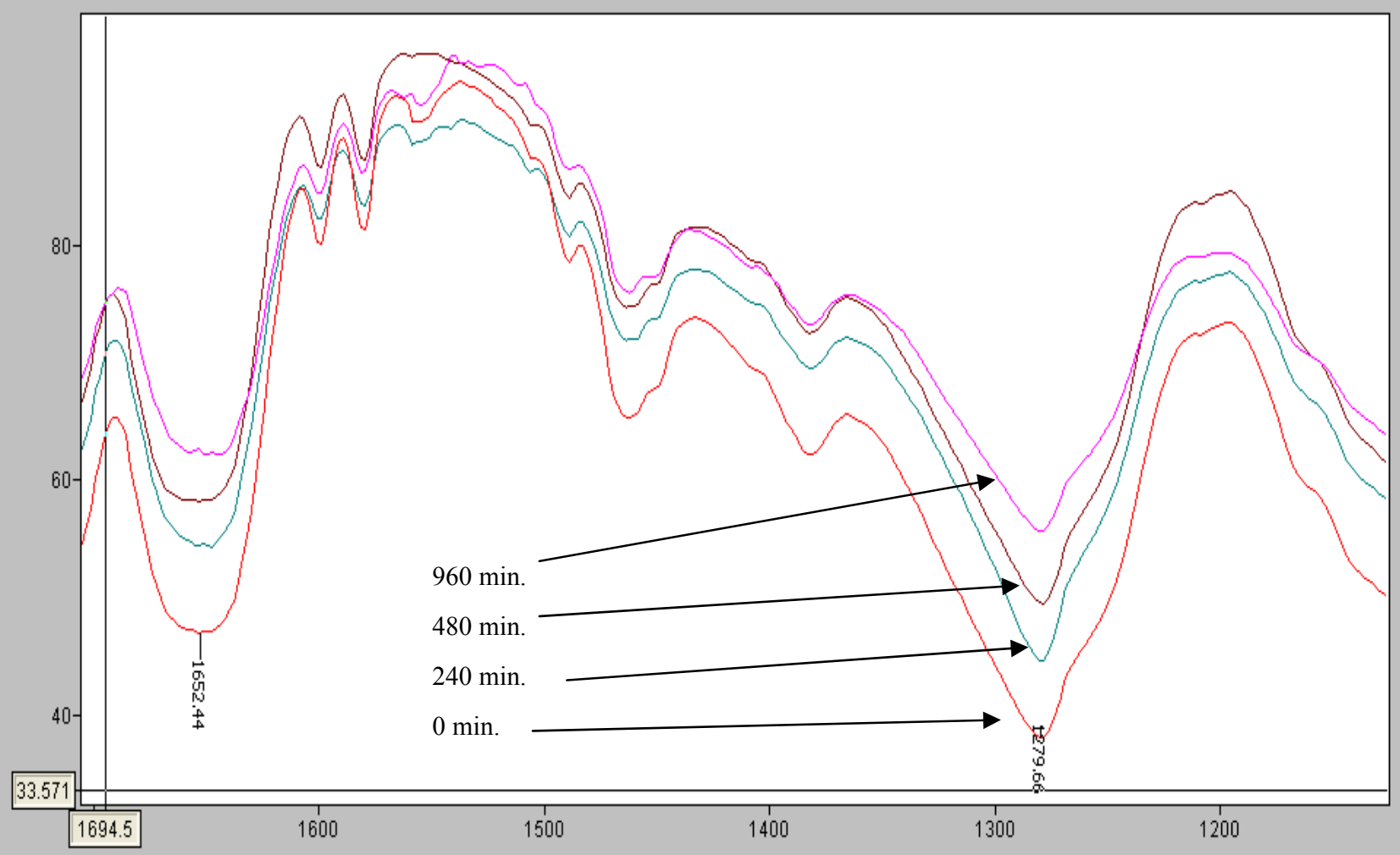

Transmittance / Wavenumber (cm-1)

Figure 4 a) 


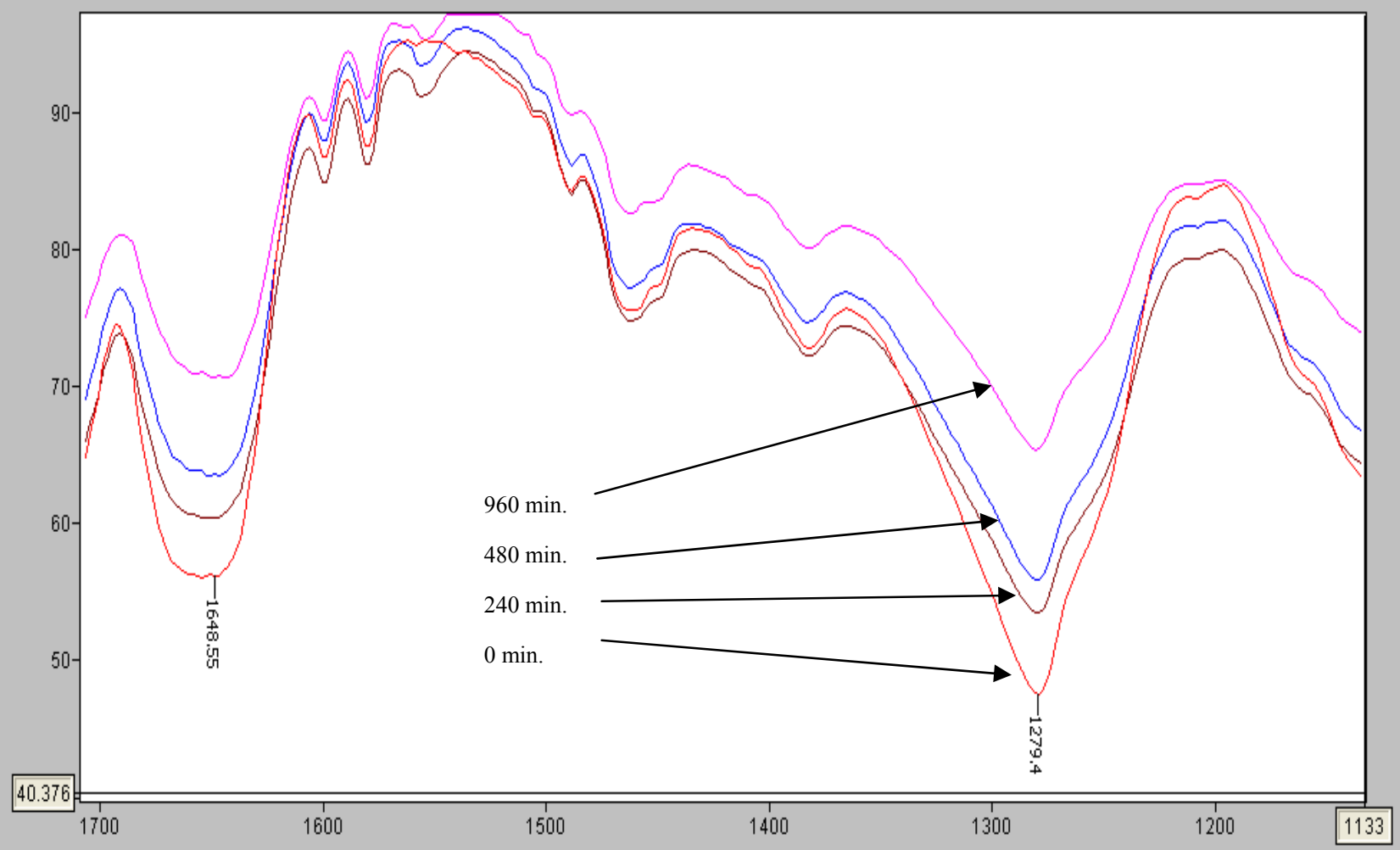

Transmittance / Wavenumber ( $\mathrm{cm}-1)$

Figure 4 b)

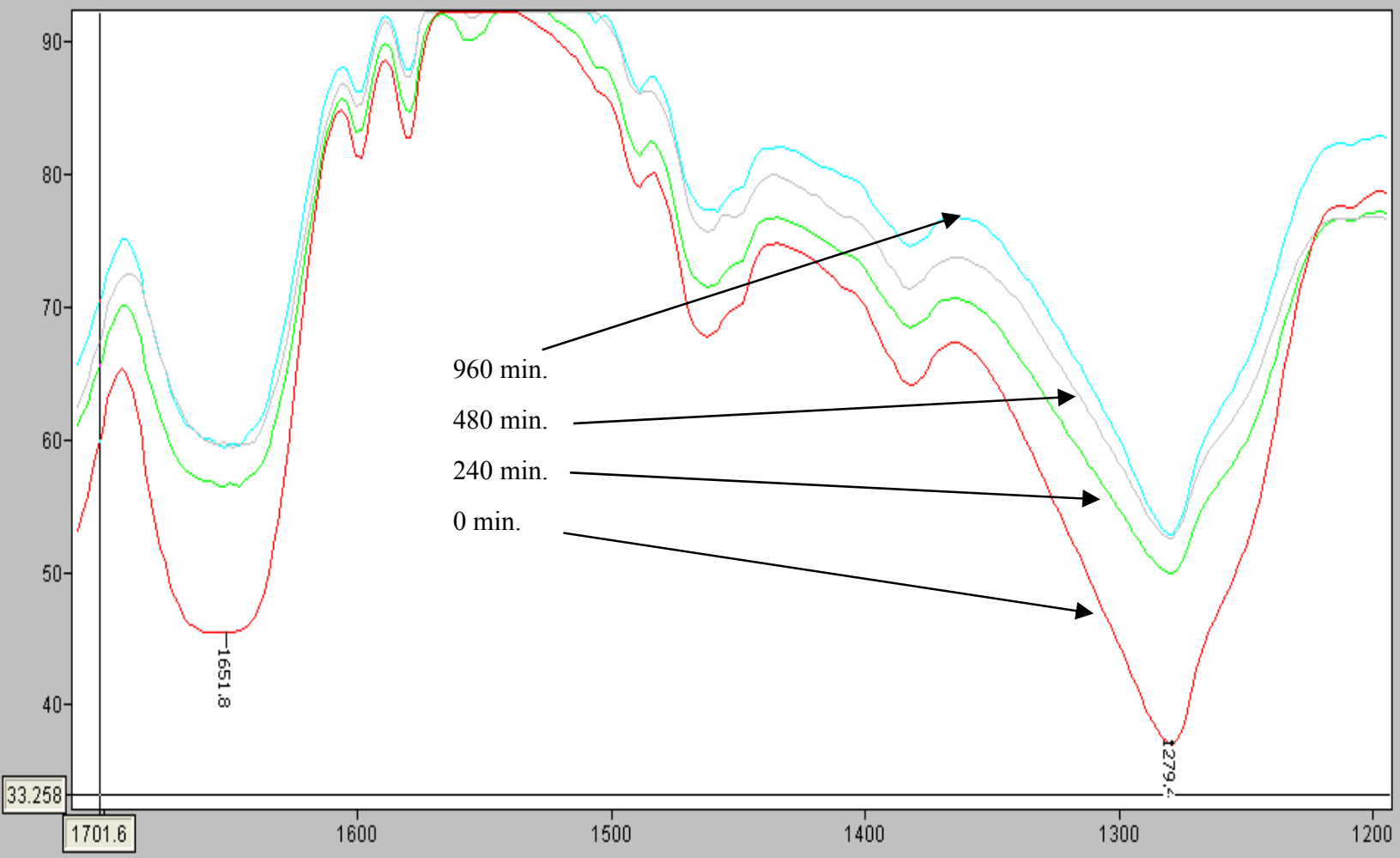

Transmittance / Wavenumber ( $\mathrm{cm}-1)$

Figure $4 \mathrm{c}$ ) 


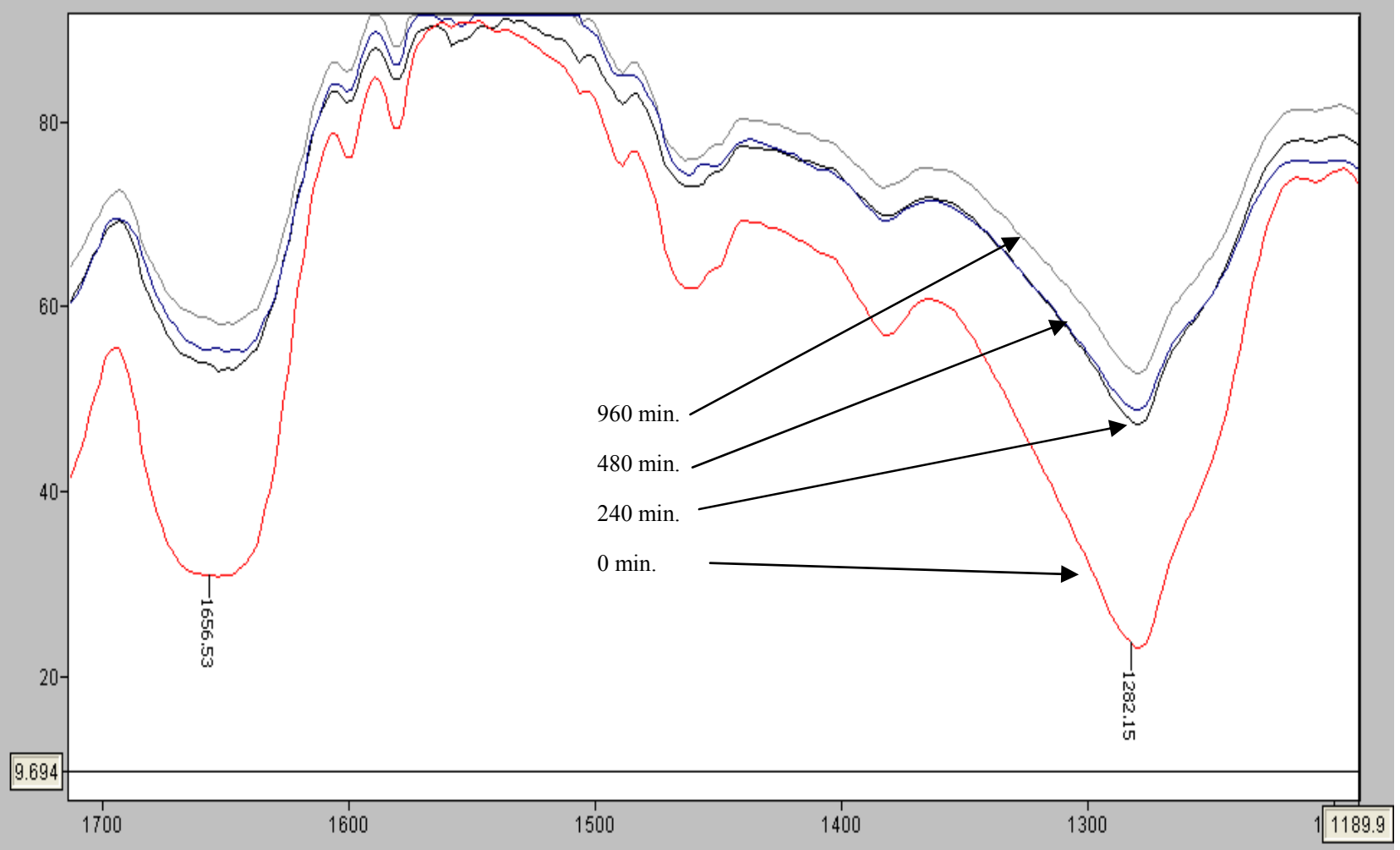

Transmittance / Wavenumber (cm-1)

Figure 4 d)

Figure 4. Comparative FTIR spectrum of the nitric group for:

a) sample I, b) sample II, c) sample III, d) sample IV.

For the sake of comparison and defining the impact of UV radiation on the process of degradation of nitrodope samples, the surfaces of those characteristic IR bands of the nitric group have been calculated. The obtained values are shown in Table 8 .

Table 8. Surfaces of the characteristic IR bands of a nitro group for nitrodope samples with different contents of nitrocellulose.

\begin{tabular}{|c|c|c|c|c|c|c|c|c|}
\hline \multirow{2}{*}{$\begin{array}{l}\text { Action } \\
\text { time of } \\
\text { UV- rays } \\
\text { (min.) }\end{array}$} & \multicolumn{2}{|c|}{ Sample I } & \multicolumn{2}{c|}{ Sample II } & \multicolumn{2}{c|}{ Sample III } & \multicolumn{2}{|c|}{ Sample IV } \\
\cline { 2 - 10 } & $v_{\mathrm{as}\left(1660 \mathrm{~cm}^{-1}\right)}$ & $v_{\mathrm{s}\left(1280 \mathrm{~cm}^{-1}\right)}$ & $v_{\mathrm{as}\left(1660 \mathrm{~m}^{-1}\right)}$ & $v_{\mathrm{s}\left(1280 \mathrm{~cm}^{-1}\right)}$ & $v_{\mathrm{as}\left(1660 \mathrm{~cm}^{-1}\right)}$ & $v_{\mathrm{s}\left(1280 \mathrm{~cm}^{-1}\right)}$ & $v_{\mathrm{as}\left(1660 \mathrm{~cm}^{-1}\right)}$ & $v_{\mathrm{s}\left(1280 \mathrm{~cm}^{-1}\right)}$ \\
\hline 0 & 38.28 & 66.70 & 41.86 & 67.86 & 45.60 & 68.44 & 47.53 & 70.21 \\
\hline 240 & 37.41 & 65.55 & 40.05 & 66.70 & 43.71 & 67.86 & 46.56 & 68.15 \\
\hline 480 & 36.55 & 64.41 & 39.16 & 65.55 & 42.78 & 66.70 & 44.65 & 67.57 \\
\hline 960 & 35.28 & 63.84 & 38.28 & 64.98 & 41.86 & 65.55 & 43.24 & 66.12 \\
\hline
\end{tabular}

As observable from the calculated values for the surfaces of the bands with valence vibrations of the nitric group, the bands' surfaces grow less after the action of UV-rays, and to the largest extent in sample IV. The surfaces of the bands become increasingly reduced as the the time period of UV 
radiation grows longer, the greatest reduction being in sample IV, which indicates that the highest degree of degradation occurs in sample IV [12;13].

\section{Conclusion}

The monitoring of the changes of the physical-mechanical properties of nitrodopes, through different mass percentages of nitrocellulose, leads to the conclusion that the FTIR spectroscopy has proved to be a proper method in studying the characteristic alterations that occur in the process of nitrodope degradation. The degree of the nitrodope degradation has been examined by determining the surfaces of the characteristic bands of the nitric group using the original Win-Bomem\&Easy software. The length of the action of UV-rays on the samples, over the time periods of 0, 240, 480 and 960 minutes, directly affects the spectrum appearance of the same sample before and after the exposure. The longer the action time of the UV-rays and also the higher a mass percentage of nitrocellulose in nitrodope are, the higher the degree of degradation grows, that is, the surfaces of the bands typical of the nitric group become smaller. The hardness lessening of the formed nitrodopes confirms that the degradation increases with the growth of the nitrocellulose percentage in the nitrodope. The values of the limiting viscosity number $[\eta]$ and the mean molar mass $M_{\eta}$ decrease with the prolongation of the action period of UV-rays. This decrease of the mean molar mass value, upon the degradation, also results in weakening of the adhesion between the dry nitrodope films and the surface. Likewise, depending on the proportion of other components in the prescriptions composition for colorless nitrodope, the programming of the nitrodope mass percentage is required in order to achieve the longterm stability and optimal mechanical properties.

\section{Acknowledgements}

We gratefully acknowledge the financial support from the Ministry of Science, Technology and Development, Republic of Serbia (grant number ON 142039, BTN 351002.B and BTN 351004.B) for support of the work.

\section{References}

1. Hon, D.N-S.; Tang, L.G. Photodegradation of Cellulose Nitrate. Polymer Photochemistry 1986, 7 , 299-310.

2. Selwitz, C.M. Cellulose nitrate in conservation. Research in Conservation, The Getty Conservation Institute: United States of America, 1988.

3. Hon, D.N-S.; Tang, L.G. Atmospheric Oxidation of Cellulose Nitrate Lacquers. Journal of Coatings Technology 1988, 60, 73-76.

4. Clark, D.T.; Fowler, A.; Steven, P.J. Application of Modern Analytical Techniques to the Investigation of Cellulose Nitrates. Reviews in Macromolecular Chemistry 1982, C23(2), 217 246.

5. Rogov, Y.N.; Smirnov, L.P.; Leksina, L.N.; Ushakova, V.S. Change in molecular characteristics during thermal decomposition of cellulosenitrate. Polymer Science U.S.S.R. 1989, 31(2), 387-394. 
6. Ng, F.M.F.; Yu, K.N. X-ray irradiation induced degradation of cellulose nitrate. Materials Chemistry and Physics 2006, 100(1), 38-40.

7. Clark, D.T.; Stephenson, P.J. ESCA study of the surfacechemistry of cellulosenitrates and double based propellants, with particular reference to their degradation in ultra-violet light. Polymer Degradation and Stability 1982, 4(3), 185-193.

8. Lawton, T.S.; Nason. H.K. Effect of Ultraviolet Light on Cellulose Acetate and Nitrate. Industrial and Engineering Chemistry 1944, 36, 1128-1130.

9. Cohen, S.M.; Young, R.H.; Marthart, A.H. Transparent Ultraviolet-Barrier Coatings. Journal of Polymer Science 1971, A-19(11), 3263.

10. Dehant, I.; Danc, R.; Kimmer, V. Инфраkрасная спектроскопия полимеров, Мосkва, Издательство «Химия» 1976, 408.

11. Villeneuve, S.; Wolf, J.-P.; Bolle, T.; Macor, G.; Stipan, G.; Kunz, M.; Bauer, M. New priming technology adhesion performance of UV-curable caotings on plastic. JCT Coatings Technology 2004, 1(5), 46-51.

12. Rogov, Y.N.; Smirnov,L.P.; Leksina, L.N.; Ushakova, V.S. Change in molecular characteristics during thermal decomposition of cellulosenitrate. Polymer Science U.S.S.R. 1989, 31(2), 387-394.

13. Yip, C.W.Y.; Nikezic, D.; Ho, J.P.Y.; Yu, K.N. Chemical etching characteristics for cellulose nitrate. Materials Chemistry and Physics 2006, 95, 307-312.

C 2007 by MDPI (http://www.mdpi.org). Reproduction is permitted for noncommercial purposes. 